\title{
BMJ Open Passive versus active intra-abdominal drainage following pancreatic resection: does a superior drainage system exist? A protocol for systematic review
}

\author{
Lily Park, ${ }^{\oplus 1}$ Laura Baker, ${ }^{2}$ Heather Smith, ${ }^{2}$ Alexandra Davies, ${ }^{3}$ Jad Abou Khalil,, ${ }^{4,5}$ \\ Guillaume Martel, ${ }^{4,5}$ Fady Balaa, ${ }^{4}$ Kimberly A Bertens ${ }^{4,5}$
}

To cite: Park L, Baker L, Smith $\mathrm{H}$, et al. Passive versus active intra-abdominal drainage following pancreatic resection: does a superior drainage system exist? A protocol for systematic review. BMJ Open 2019;9:e031319. doi:10.1136/ bmjopen-2019-031319

- Prepublication history and additional material for this paper are available online. To view these files, please visit the journal online (http://dx.doi. org/10.1136/bmjopen-2019031319).

Received 28 April 2019 Revised 17 August 2019 Accepted 02 September 2019

\section{Check for updates}

(c) Author(s) (or their employer(s)) 2019. Re-use permitted under CC BY-NC. No commercial re-use. See rights and permissions. Published by BMJ.

${ }^{1}$ School of Medicine, University of Ottawa, Ottawa, Ontario, Canada

${ }^{2}$ General Surgery, The Ottawa Hospital, Ottawa, Ontario,

Canada

${ }^{3}$ Library Services, The 0ttawa Hospital, Ottawa, Ontario,

Canada

${ }^{4}$ Hepatopancreaticobiliary Surgery, Department of Surgery, The Ottawa Hospital, Ottawa, Ontario, Canada

${ }^{5}$ Clinical Epidemiology, Ottawa Hospital Research Institute, Ottawa, Ontario, Canada

Correspondence to Dr Kimberly A Bertens; kbertens@toh.ca

\section{ABSTRACT}

Background Clinically relevant postoperative pancreatic fistula (CR-POPF) is the most common cause of major morbidity following pancreatic resection. Intra-abdominal drains are frequently positioned adjacent to the pancreatic anastomosis or transection margin at the time of surgery to aid in detection and management of CR-POPF. Drains can either evacuate fluid by passive gravity (PG) or be attached to a closed suction (CS) system using negative pressure. There is controversy as to whether one of these two systems is superior. The objective of this review is to identify and compare the incidence of adverse events (AEs) and resource utilisation associated with PG and CS drainage following pancreatic resections.

Methods and analysis MEDLINE, EMBASE, CINAHL and Cochrane Central Registry of Controlled Trials will be searched from inception to April 2019, to identify interventional and observational studies comparing PG and CS drains following pancreatic resection. The primary outcome is POPF as defined by the International Study Group for Pancreatic Fistula in 2017. Secondary outcomes include postoperative $\mathrm{AE}$, resource utilisation (length of stay, return to emergency department, readmission and reintervention), time to drain removal and quality of life. Study selection, data extraction and risk of bias assessment will be performed independently, by two reviewers. A meta-analysis will be conducted if deemed statistically appropriate. Subgroup analysis by study design will be performed. Study heterogeneity will be calculated with the $\chi^{2}$ test and reported as $I^{2}$ statistics. Statistical analyses will be conducted and displayed using RevMan V.5.3

Ethics and dissemination Ethics approval is not required. The results of this study will be submitted to relevant conferences for presentation and peer-reviewed journals for publication.

PROSPERO registration number CRD42019123647.

\section{INTRODUCTION}

Pancreatic resection is commonly performed for benign and malignant diseases of the pancreas, duodenum and distal biliary tree. ${ }^{1}$ Despite substantial improvements in perioperative mortality, morbidity remains high, approaching 50\%. ${ }^{2}{ }^{3}$ Postoperative
Strengths and limitations of this study

- This protocol provides transparency to the process of systematic review development and accountability for the authors such that bias is minimised.

- This systematic review would investigate whether there are outcome differences between closed suction (CS) and passive gravity (PG) drainage following pancreatic resections, and which other factors could potentially impact these outcomes.

- Highest level of evidence for informed decision-making would be made available from this systematic review of randomised controlled trials (RCTs), nonRCTs and high-quality observational studies

- May be limited by lack of primary studies available at this time exploring PG versus CS drainage systems

pancreatic fistula (POPF), is the largest contributor to major morbidity following pancreatic resection, with reported incidence ranging between $10 \%$ and $35 \% \cdot{ }^{3-5} \mathrm{POPF}$ has been objectively defined as drainage of amylase-rich fluid from a surgically placed drain, on or after, postoperative day $3 .{ }^{4}$ Leakage of pancreatic fluid can predispose patients to a myriad of related morbidity such as surgical site infection (SSI), post pancreatectomy haemorrhage (PPH), delayed gastric emptying, multisystem organ failure and even death. ${ }^{36}$ In addition, POPF contributes to significant economic burden, as healthcare cost can nearly double in the setting of a POPF. ${ }^{78}$

In 2005, the International Study Group for Pancreatic Fistula (ISGPF) defined POPF using an objective classification system. ${ }^{45}$ The severity of pancreatic fistula ranges from those without deviation from routine postoperative course (grade A), to those that are life-threatening (grade C). ${ }^{5}$ Since grade A leaks do not impact outcome or management, in 2017, the ISGPF published an updated classification 
system in which grade A leaks are no longer classified as POPF, and instead referred to as a biochemical leak. Grades B and C POPF, by definition pose deviations from the routine postoperative course and therefore, both fall under the umbrella term 'clinically relevant' (CR)-POPF. ${ }^{5}$

Extensive investigation has been devoted to confronting the problem of pancreatic fistulas following pancreatic surgery. Various technical modifications to the pancreatic anastomosis, anastomotic reinforcement with fibrin glue, placement of internal and external stents as well as somatostatin analogues have all been investigated as possible mitigating factors for POPF. ${ }^{3}$ Despite substantial efforts to reduce the incidence of CR-POPF, it remains stable between $20 \%$ and $35 \% .{ }^{5910}$

The ability of the type of surgically placed drainage system to influence the development and severity of CR-POPF has yet to be robustly investigated. Surgically placed, intra-abdominal drains are frequently placed following pancreatic resection, for the purpose of evacuating pancreatic effluent, if a leak occurs. Drains are typically positioned with one end in close proximity to the pancreatic anastomosis or parenchymal transection margin, while the other end transverses the abdominal wall and is attached to a device that serves as a reservoir for drained fluid. This reservoir can either be collapsed, generating a negative pressure, closed suction (CS) environment, or function as a vessel, allowing fluid to drain by passive gravity (PG). The CS systems generate pressure measured at $-150 \mathrm{~mm} \mathrm{Hg}$ when the bulb is fully decompressed and up to $-200 \mathrm{~mm} \mathrm{Hg}$ when the drain is stripped. ${ }^{11} 12$ As such, there is a theoretical belief that the high-pressure gradient generated by CS drainage has the potential to promote the development of a POPF. ${ }^{11}{ }^{12}$ Conversely, it has also been postulated that CS drainage promotes improved drainage and collapse of the surgical dead space, thereby decreasing the severity of a leak if it does occur. ${ }^{11}{ }^{12}$ Despite anecdotal beliefs on the superiority of each type of drainage system, to date, few studies directly compare PG with CS and both are used depending on a surgeon's idiosyncratic preference.

\section{Objective}

The objective of this review is to compare the incidence of CR-POPF, other adverse events (AEs), and resource utilisation between CS and PG drainage following pancreatic resections. Results of this review will help inform future practice or need for further investigation.

\section{METHODS}

The Preferred Reporting Items for Systematic Review and Meta-analysis Protocols (PRISMA-P) checklist guideline will be followed to achieve the highest standard in reporting (online supplementary appendix 1). ${ }^{13}$ A PRISMA flow diagram will be used to display the screening strategy. The protocol was registered with the PROSPERO International Prospective Register of Systematic Reviews on 26 February 2019.
Any amendments made to the current protocol will be published using a protocol addendum. This will be accompanied by the date and rationale for the reported amendment, with the final manuscript.

\section{Eligibility criteria}

Study designs

Published interventional and observational studies comparing the incidence of postoperative AEs in patients who had a PG or CS drain placed at the time of pancreatic resection will be considered for inclusion.

\section{Population}

Adult patients, 18 years of age or older, who have undergone elective pancreatic resection for any indication will be considered for inclusion. Pancreatic resection includes pancreaticoduodenectomy, distal pancreatectomy, central pancreatectomy and pancreatic enucleation. Necrosectomy in the setting of pancreatitis will be excluded, as this represents a group of patients distinctly different from those undergoing elective pancreatic resection. Patients undergoing total pancreatectomy will also be excluded given that there is no pancreatic parenchyma remaining, and therefore, no chance of POPF. Lastly, drains must be placed at the time of surgery to be considered eligible.

\section{Comparators}

The two comparator groups are intra-abdominal drains, placed at the time of surgery, and connected to PG or CS drainage systems. A PG surgical drainage system is defined as a drain that maintains a pathway for fluid to follow from the surgical site by gravity, which is connected to reservoir maintained at atmospheric pressure. A closed negative pressure (CS) surgical drainage system is defined as a drain connected to a collapsible reservoir, which generates a negative pressure relative to atmospheric pressure.

Drains managed with continuous irrigation will not be considered in the proposed review. Flushing of minimal fluid with the intention of maintaining drain patency is an acceptable intervention.

\section{Outcomes}

The primary outcome of interest is development of a CR-POPF, as defined by the ISGPF 2017 definition. ${ }^{45}$ Incidence of biochemical leak (formerly defined as grade A leak) will also be assessed.

Secondary outcomes of interest include postoperative AEs, resource utilisation and time to drain removal. Postoperative AEs of interest include occurrence of morbidity as defined by the Clavien-Dindo Classification system, ${ }^{14}$ SSI (superficial, deep or organ space infection), sepsis, postoperative pancreatitis, $\mathrm{PPH}$, biliary leak, intestinal leak, requirements for percutaneous drainage, reoperation and death. Resource utilisation will also be assessed by comparing: length of stay, intensive care unit (ICU) admission and associated length of stay, return to the emergency department, readmission to hospital and requirement of home care following discharge. In 
addition, time to drain removal, presence of drain at discharge and quality of life outcomes will be examined.

\section{Study type}

All studies (both observational and interventional) meeting inclusion criteria will be considered, regardless of study duration, language of publication, sample size or geographic location. Only published data will be considered for inclusion. For studies published as abstract only, the authors will be contacted for additional data, and included in the analysis if inclusion criteria are met.

\section{Search strategy}

A systematic search of electronic databases will be performed to identify all relevant studies investigating differences in outcomes for passive versus active intra-abdominal drainage systems following pancreatic resections. A reference librarian was consulted to assist with the development of database specific search strategies (online supplementary appendix 2). Medical Subject Headings and keywords were used to search the following themes: pancreatic surgery and drains.

The search strategy will be applied to the following databases: MEDLINE (PubMed, PubMed in Process and Ovid), EMBASE, CINAHL and Cochrane Central Registry of Controlled Trials. References of included manuscripts will be reviewed to identify additional studies of relevance. The databases will be queried from inception to 1 August 2019.

\section{Study selection}

Articles identified through the search strategy will be collated and exported to a citation manager (Covidence Systematic Review Software, Veritas Health Innovation, Melbourne, Australia ${ }^{15}$ ) for screening by three of the authors (LP, LB and HS). Abstracts and titles will be screened through the Covidence platform, ${ }^{15}$ independently and in duplicate. Eligible studies will then be advanced to full-text review, where they will also be assessed in triplicate for inclusion (LP, LB and HS). Any disagreement regarding relevancy will be resolved by the senior author (KB). Reason for study exclusion will be documented and presented in the PRISMA flow diagram for study screening.

\section{Data extraction}

Data extraction for included studies will be conducted independently, in triplicate, by three reviewers (LP, LB and HS) using a standardised electronic data extraction form. The data extraction form will be piloted by all reviewers. The following information will be extracted from each article: study identifiers (title, authors, journal, publication date, study location(s), funding and duration of follow-up), aspects of study design (interventional vs observational, blinding, allocation concealment, duration, setting and number of centres), patient characteristics (inclusion/exclusion criteria, baseline demographics and fistula risk score,${ }^{16}{ }^{17}$ information pertaining to the intervention (location of drain placement), primary and secondary outcomes, as previously described, and information pertaining to drain duration (presence of drain at discharge, time to drain removal and details pertaining to decision-making around drain removal).

In the event data pertaining to the intervention or outcomes of interest are missing, authors will be contacted in an attempt to retrieve this information. If a response is not provided by the corresponding authors within a 1-week period, they will be contacted two additional times.

In the event studies referring to the same patient population are identified (duplicate, overlapping or companion studies), only the most comprehensive study will be included. Should this be deemed comparable between studies, the most recent study will be included.

\section{Risk of bias/quality assessment}

The risk of bias of included studies will be evaluated by two independent assessors (LP and LB). The Cochrane Collaboration's tool for assessing risk of bias in randomised controlled trials (RCTs) will be used to assess interventional trials while the Methodological Index for Non-Randomized Studies (MINORS) tool will be used for observational studies. ${ }^{18}{ }^{19}$ A MINORS Score $\geq 17$ will be considered high quality, as previously reported in literature. $^{19} 20$

\section{Data synthesis}

Descriptive summary tables of included studies, and primary and secondary outcomes will be presented. If appropriate, a direct meta-analysis will be performed for primary and secondary outcomes of both interventional and observational studies. Incidence of CR-POPF, SSI, postoperative pancreatitis, $\mathrm{PPH}$, biliary leak, intestinal leak, need for percutaneous drainage, re-operation, death, re-presentation to the ED, re-admission to the hospital and presence of drain at discharge will be analysed as dichotomous data reported with odds ratios and 95\% CIs. Hospital length of stay, ICU admission and associated length of stay and time to drain removal will be analysed as continuous data and reported as weighted mean differences with $95 \%$ CI. If the data are reported as median and range, an established calculation method by Hozo $e t a l^{21}$ will be used to translate the values into their mean and SD estimates. Due to anticipated heterogeneity between studies, a random effects model will be used to generate forest plots. A $p$ value of $<0.05$ will be considered statistically significant for all analyses.

Potential sources of clinical and methodological heterogeneity between included studies will be examined using the $\chi^{2}$ test and the $\mathrm{I}^{2}$ statistic. The thresholds for interpretation will be defined in accordance with the Cochrane Handbook for Systematic Reviews of Interventions. ${ }^{22}$

If significant heterogeneity is identified, sensitivity analysis will be performed to explore potential sources of heterogeneity. This analysis will exclude articles at high risk of bias and abstract publications (compared with full-text publications). Additionally, a planned 
subgroup analysis will be conducted by study design (interventional vs observational), if appropriate. Given that the complication profile of pancreaticoduodenectomy and distal pancreatectomy differ, only the primary outcome of CR-POPF will be considered for analysis in a pooled cohort of all pancreatic resections. The analysis of secondary outcomes will be undertaken separately by type of pancreatic resection (pancreaticoduodenectomy vs distal pancreatectomy vs other OR pancreaticoduodenectomy vs other),

RevMan V.5.3 software (Copenhagen: The Nordic Cochrane Centre, 2014) will be used to perform statistical analyses and generate figures.

\section{Patient and public involvement}

As a secondary research study, this work will not directly involve patients or the public in any stage of its development including research question design, outcome measures, study design, data collection or analysis.

\section{DISCUSSION}

Despite routine use of intra-abdominal drains following pancreatic resections, there is a lack of robust evidence to suggest superiority between CS and PG drainage systems on the development of CR-POPF. Currently, the choice between the two systems lies in surgeon preference and resource availability at individual hospitals. If a clinically significant difference exists between the two systems, the results of this review could contribute to changes in standard practice that may help reduce the morbidity rates associated with pancreatic resection.

Presently, there is a published Cochrane review that aimed to answer a related research question. ${ }^{23}$ The authors sought to assess the benefits and harms of routine abdominal drainage after pancreatic surgery, investigate the optimal time for drain removal and compare the efficacy of different types of surgical drains. ${ }^{23}$ Only one RCT by Jiang $e t a t^{24}$ was included that addressed the question of efficacy differences between drainage systems, which is relevant to this proposed systematic review. This study found no differences in morbidity or mortality at 30 days when comparing $\mathrm{CS}$ drains with continuous irrigation and PG drains. Although Jiang $e t a l^{24}$ suggested that CS drains may be associated with decreased intra-abdominal infection, wound infection and the number of additional interventions for postoperative complications, the Cochrane review deemed it to be low-quality evidence, rendering such associations uncertain. Notably, any drain used primarily for irrigation will not be considered an intervention or comparator in the present study. Therefore, the current proposed study is distinct from the Cochrane review and has potential to weigh in on the question of drainage system efficacy that remains unanswered.

In the present review, studies that involve patients undergoing any type of pancreatic resection for any indication will be considered for inclusion, which may obtain greater sample sizes to power the analyses. Subgroup analyses for sensitivity analysis will also be conducted to provide an updated review on the difference in drainage systems following pancreaticoduodenectomy exclusively, as well as other pancreatic resections (ie, distal pancreatectomy) to determine if there are differences in outcomes. In the event a meta-analysis is possible, results of this study may spearhead the development of recommendations for improved management of patients undergoing pancreatic resections. Specifically, this would provide adequate power to demonstrate significant difference in reducing CR-POPF incidence or other postoperative AEs associated with usage of different drainage systems.

Anticipated challenges include a combined data analysis between studies of considerable heterogeneity. Understanding that this review may be limited by a lack of RCTs and study heterogeneity, meta-analysis will be limited to the pooling of RCTs, quasi-randomised studies and moderate- to high-quality observational studies. Additionally, anticipation of limited published literature on this topic may necessitate an analysis of the primary outcome (CR-POPF) that pools results for all pancreatic surgeries. This may further contribute to heterogeneity since different surgery types have varying complication profiles, surgery indications and patient profiles. Although analysis of both the primary and secondary outcomes by type of pancreatic resection has been planned a priori, this may not be possible with potential paucity in literature. In such cases, conservative conclusions will be made with detailed explanation of study limitations.

A systematic review investigating the differences in outcomes between drainage systems following pancreatic resections may allow for improved morbidity and more informed recommendations on risk-management. If evidence is lacking, it will inform the need for future investigations. Considering both CS and PG drains are simple and cost-effective interventions that can be easily implemented, it would rapidly become the standard of care to use one over the other, if a true difference is found.

Acknowledgements LB is financially supported by the University of Ottawa Clinical Investigator Program and by a Canadian Graduate Scholarship. LP and HS are not funded. $A D$ is not funded for this project specifically, but is an employee of The Ottawa Hospital. JAK, GM, FB and KAB have not received funding for this project.

Contributors All authors were involved in the study conception and design. The protocol was drafted and registered with PROSPERO by LP, LB, HS and KAB. JAK, $\mathrm{GM}, \mathrm{FB}$ and $\mathrm{KAB}$ are all hepatopancreaticobiliary surgeons who provided content expertise. $A D$ is a health sciences librarian who helped develop the search strategy and provided literature search expertise. All authors reviewed and approved the final manuscript. KAB is the guarantor of the protocol.

Funding The authors have not declared a specific grant for this research from any funding agency in the public, commercial or not-for-profit sectors.

Competing interests None declared.

Patient consent for publication Not required.

Provenance and peer review Not commissioned; externally peer reviewed.

Open access This is an open access article distributed in accordance with the Creative Commons Attribution Non Commercial (CC BY-NC 4.0) license, which permits others to distribute, remix, adapt, build upon this work non-commercially, and license their derivative works on different terms, provided the original work is properly cited, appropriate credit is given, 
any changes made indicated, and the use is non-commercial. See: http:// creativecommons.org/licenses/by-nc/4.0/.

\section{REFERENCES}

1. Cameron JL, Riall TS, Coleman J, et al. One thousand consecutive pancreaticoduodenectomies. Ann Surg 2006;244:10-15.

2. Mahvi DA, Pak LM, Urman RD, et al. Discharge destination following pancreaticoduodenectomy: a NSQIP analysis of predictive factors and post-discharge outcomes. Am J Surg 2019;218:342-8.

3. Nahm CB, Connor SJ, Samra JS, et al. Postoperative pancreatic fistula: a review of traditional and emerging concepts. Clin Exp Gastroenterol 2018;11:105-18.

4. Bassi C, Dervenis C, Butturini G, et al. Postoperative pancreatic fistula: an international Study Group (ISGPF) definition. Surgery 2005;138:8-13.

5. Bassi C, Marchegiani G, Dervenis C, et al. The 2016 update of the International Study Group (ISGPS) definition and grading of postoperative pancreatic fistula: 11 years after. Surgery 2017;161:584-91.

6. Vin Y, Sima CS, Getrajdman Gl, et al. Management and outcomes of postpancreatectomy fistula, leak, and abscess: results of 908 patients resected at a single institution between 2000 and 2005. J Am Coll Surg 2008;207:490-8.

7. Ma LW, Dominguez-Rosado I, Gennarelli RL, et al. The cost of postoperative pancreatic fistula versus the cost of pasireotide: results from a prospective randomized trial. Ann Surg 2017;265:11-16.

8. Čečka F, Jon B, Čermáková E, et al. Impact of postoperative complications on clinical and economic consequences in pancreatic surgery. Ann Surg Treat Res 2016;90:21-8.

9. Bassi C, Butturini G, Molinari E, et al. Pancreatic fistula rate after pancreatic resection. The importance of definitions. Dig Surg 2004;21:54-9.

10. Bassi C, Buchler MW, Fingerhut A, et al. Predictive factors for postoperative pancreatic fistula. Ann Surg 2015;261:e99.

11. Whitson BA, Richardson E, laizzo PA, et al. Not every bulb is a rose: a functional comparison of bulb suction devices. J Surg Res 2009;156:270-3.
12. Grobmyer SR, Graham D, Brennan MF, et al. High-Pressure gradients generated by Closed-Suction surgical drainage systems. Surg Infect 2002;3:245-9.

13. Moher D, Shamseer L, Clarke M, et al. Preferred reporting items for systematic review and meta-analysis protocols (PRISMA-P) 2015 statement. Syst Rev 2015;4:1.

14. Dindo D, Demartines N, Clavien P-A. Classification of surgical complications. Ann Surg 2004;240:205-13.

15. Veritas Health Innovation. Covidence systematic review software [Internet]. Melbourne, Australia. Available: www.covidence.org

16. Callery MP, Pratt WB, Kent TS, et al. A prospectively validated clinical risk score accurately predicts pancreatic fistula after pancreatoduodenectomy. J Am Coll Surg 2013;216:1-14.

17. Miller BC, Christein JD, Behrman SW, et al. A multiinstitutional external validation of the fistula risk score for pancreatoduodenectomy. J Gastrointest Surg 2014;18:172-80.

18. Higgins JPT, Altman DG, Gøtzsche PC, et al. The Cochrane collaboration's tool for assessing risk of bias in randomised trials. BMJ 2011;343:d5928.

19. Slim K, Nini E, Forestier D, et al. Methodological index for nonrandomized studies (minors): development and validation of a new instrument. ANZ J Surg 2003;73:712-6

20. Abraham NS, Byrne CJ, Young JM, et al. Meta-Analysis of welldesigned nonrandomized comparative studies of surgical procedures is as good as randomized controlled trials. J Clin Epidemiol 2010;63:238-45.

21. Hozo SP, Djulbegovic B, Hozo I. Estimating the mean and variance from the median, range, and the size of a sample. BMC Med Res Methodol 2005;5:13.

22. Higgins J, Green S. Cochrane Handbook for Systematic Reviews of Interventions Version 5.1.0 [updated March 2011] [Internet]. The Cochrane Collaboration, 2011. Available: www.handbook.cochrane. org

23. Zhang W, He S, Cheng Y. Prophylactic abdominal drainage for pancreatic surgery. Cochrane Database of Systematic Reviews [Internet], 2018. Available: https://www.readcube.com/articles/10. 1002/14651858.CD010583.pub4

24. Jiang $\mathrm{H}$, Liu $\mathrm{N}$, Zhang $\mathrm{M}$, et al. A randomized trial on the efficacy of prophylactic active drainage in prevention of complications after pancreaticoduodenectomy. Scand J Surg 2016;105:215-22. 